\title{
On the exciton model for ion-beam damage: The example of $\mathrm{TiO}_{2}$
}

\author{
A. Rivera , M.L. Crespillo , J. Olivares ，R. Sanz , J. Jensen , F. Agulló-López \\ Instituto de Microelectrónica de Madid, (CNM-CSIC), Isaac Newton 8, E-28760 Tres Cantos, Spain \\ Centro de Microanálisis de Materiales (CMAM). Universidad Autónoma de Madrid (UAM). Cantoblanco, E-28049 Madrid, Spain \\ instituto de Optica, Consejo Superior de investigaciones Científicas (CSIC). C/Serrono 121, E-28006 Madrid, Spain \\ Instituto de Ciencia de Materiales de Madrid (ICMM-CSIC). Cantoblanco, E-28049 Madrid, Spain \\ Thin Firm Physics Division, Department of Physics, Chemistry and Biology - IFM, Linköping University, SE 581 83, Linköping, Sweden \\ Departamento de Física de Materiales, Universidad Autónoma de Madird (UAM), Cantobianco, E-28049 Madrid, Spain
}

Keywords:

Damage

Excitons

Ion beams

Rutile

RBS/C

Channelling amorphization threshold

\section{A B S T R A C T}

The non-radiative exciton decay model recently developed to account for swift-ion-beam damage to $\mathrm{LiNbO}_{3}$ is, here, discussed within a general physical perspective, taking previous work on alkali halides as a reference. Some general rules for the validity of excitonic models have been put forward, allowing one to predict the irradiation behaviour of other materials. As a new example of application, some preliminary data on the generation and growth of uniform amorphous layers induced by irradiation with $\mathrm{Br}$ at $13 \mathrm{MeV}$ and $25 \mathrm{MeV}$ have been performed on rutile $\left(\mathrm{TiO}_{2}\right)$. In addition sub-threshold irradiations with Br ions at $9 \mathrm{MeV}$ have been carried out. Defects generation is observed as a result. This elTect is explained with the exciton model. Experiments are in the electronic excitation regime and use moderate fluences in the range of $6 \times 10^{12}$ to $1.5 \times 10^{14} \mathrm{~cm}^{2}$. The results show similar features to those found for $\mathrm{LiNbO}_{3}$ and are, in principle, consistent with a non-radiative exciton decay model.

\section{Introduction}

The structural damage to a crystal lattice caused by the electronic excitation associated to irradiation with swift-heavy ions (SHI) remains an unsolved problem. This can be formulated as follows: how the high energy deposited in electronic excitation is transferred to the ionic lattice and leads to bond breaking and defect generation? The lack of this basic understanding may limit the application of SHI irradiation in the emerging fields of nano-electronics and nano-photonics. Essentially two different approaches or strategies have been explored, namely those focusing on the role of massive excitation, as in the Coulomb explosion model [1], or those focused on the high temperatures reached in the thermal spike generated by the ion impact (thermal spike models [2-5]). The thermal spike model extensively developed by Toulemonde and co-workers [2-4] belongs to the second approach and it has turned out to be quite satisfactory to account for a number of experimental features both in dielectrics and semiconductors. In particular, the model is able to predict the threshold value of the stopping power required to cause amorphization and even the size of the amorphized areas caused by each ion impact (tracks). How- ever, in this model the electronic excitation is not explicitly considered and only appears as the source for the energy loss in the material. Moreover, it cannot account for some other relevant features observed for $\mathrm{LiNbO}_{3}$ and other materials, namely, the damage observed under sub-threshold conditions, the halo appearing around the anorphous core of a track and the cumulative character of the domage. Recently, a new model [6,7] (based on an initial suggestion by ltoh [8]) has been put forward. It combines the thermal spike approach with a non-radiative exciton decay and satisfactorily explains many of those damage features for $\mathrm{LiNbO}_{3}$. However, the application of the model to other dielectric crystals should be explored so that the generality and limitations of the excitonic approach could be properly evaluated.

The purpose of this work is to discuss the exciton model within a more general perspective taking as a reference the well-documented case of alkali halides that serves as a comparative example [9-12]. On the basis of this information some general guidelines will be offered that determine the feasibility of exciton models in dielectric or semiconductor materials. $\mathrm{TiO}_{2}$, presenting two common polymorphs, rutile and anatase, appears as an interesting dielectric material having a variety of industrial and technological applications [13]. A few swift-ion irradiation experiments in the isolated track regime have been already performed on rutile in relation to nano-patterning and nano-photonics [14-18]. Here, we will describe preliminary ion-beam irradiation experiments on this material in the regime of moderate fluences, where uniform amorphous layers are generated. Although the work should be 
further pursued and more information is needed on defects and excitons, the data are consistent with an exciton model. In particular, the defect evolution in the sub-threshold regime (before full amorphous tracks are created) is quantitatively described with the exciton model, while other models fail to give a satisfactory description. This work provides an additional example of application of the exciton model to oxide materials.

\section{Physical bases of the exciton model. General criteria for applicability}

Alkali halides, having a wide electronic energy gap, constitute a classical and paradigmatic example of excitonic models for ionization damage and provide an adequate ground to discuss the rules that should apply to other more complex situations. After an extensive research effort it was concluded that Frenkel pairs can be generated by purely ionizing radiation (UV light, X-rays and gamma rays) through de-excitation of self-trapped (localized) excitons. The core of those excitons is constituted by a self-trapped hole at a $\mathrm{X}^{-}$halogen site that forms a $\mathrm{X}_{2}^{-}$molecule after relaxation and binding to a first-neighbour $\mathrm{X}^{-}$ion $[9,12]$. The self-trapped hole then acts as a recombination site for its electron partner through a coupled pair (exciton). Although the physics is complex, in brief, the luminescence emission of the exciton states operates in competition with the non-radiative defect-formation channel leading to the generation of a Frenkel pair. The primary efficiency for defect-formation has been measured as a function of temperature and can be explained by a peculiar structure of the potential energy curves leading to lattice recovery [10]. In other words, the efficiency is determined by an Arrhenius-type survival probability. This idea summarizes some key features of the exciton model, namely the occurrence of a non-radiative decay channel, which competes with the radiative emission. The non-radiative decay channel operates by overcoming a certain energy barrier in the relaxation profile leading to defect-formation.

On the basis of the information gathered for alkali halides one can now formulate some general rules to discuss exciton models in a more general context. In order to assure an efficient defect generation process from the non-radiative decay of the excitation energy stored at the exciton one should propose a few general criteria [11]:

(a) The excitation energy has to be well localized in a given lattice site so that the decay energy is transferred to a few atomic bonds, e.g. by forming a localized or trapped exciton. In fact, the occurrence of carrier as well as exciton self-trapping is a common feature $[12,19]$ in many dielectric (strongly polarizable) crystals, including halides, dihalides and binary or ternary transition metal oxides (e.g. MnO, $\mathrm{NiO}, \mathrm{SrTiO}_{3}$ and $\mathrm{BaTiO}_{3}$ Electron or/and hole self-trapping is, often, a pre-requirement for exciton localization.

(b) One of the non-radiative de-excitation channels should lead to lattice reorganization, i.e., bond breaking and point defect-formation. This condition demands that the exciton energy is high enough to cause such a disorder. In other words, the energy stored in one single excitation (exciton) must be larger than the energy required to break one bond. In general, the operative channel may be thermally activated and requires the overcoming of a certain energy barrier. The energy necessary to overcome the barrier stems from the thermal spike, i.e., the energy deposited as heat

(c) The lifetime of the bond breaking channel has to be short enough in comparison with that of other competing relaxation channels to assure the generation of point defects. This requires that the thermal energy barrier is low enough. (d) The non-radiative de-excitation channel should represent a significant fraction of the total decay rate.

(e) The defects generated have to be separated far enough to avoid recombination and assure their survival.

\section{Swift-ion-beam damage to $\mathrm{TiO}_{2}$ (rutile)}

$\mathrm{TiO}_{2}$ (rutile) is a positive birrefringent oxide with very high refractive indices in the visible: $n_{0}=2.616$ and $n_{\mathrm{e}}=2.903$ at $\lambda=589 \mathrm{~nm}$. It presents a centrosymmetric tetragonal structure (space group, $\mathrm{P} 4_{2} / \mathrm{mnm}, D_{4 h}^{14}$, which contains six atoms per unit cell [20]. This structure can be seen as a stacking of oxygen octahedra centered on titanium atoms (Fig. 1), with lattice parameters $a=4.594 \AA$ and $c=2.959 \AA$ (mass density $\rho=4.25 \mathrm{~g} / \mathrm{cm}^{3}$ ) and a direct electronic energy gap of $3.05 \mathrm{eV}$ [21]. It may be an interesting candidate to explore the application of an exciton model. Some fundamental optical spectroscopy studies have been carried out on both rutile and anatase [22-25]. Although the situation may be not definitely settled, it appears that self-trapped excitons exist, indeed, in anatase but there is not evidence for rutile. Anyhow, other types of localized excitons may occur in rutile and also local phase changes during irradiation may lead to mixed anatase-rutile phases.

\subsection{Experimental data}

Rutile single crystals $\mathrm{TiO}_{2}\left(5 \times 5 \mathrm{~mm}^{2}\right)$ (Crystal $\left.\mathrm{Gmbh}\right)$ with a

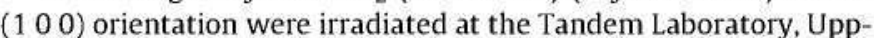
sala University and at the $5 \mathrm{MV}$-Tandetrom accelerator at Centro de Microanálisis de Materiales (CMAM-UAM, Madrid) with $\mathrm{Br}$ ions at 9, 13 and $25 \mathrm{MeV}$. At Uppsala University the samples were irradiated with $\mathrm{Br} 13$ and $25 \mathrm{MeV}$, while the irradiation experiments with $\mathrm{Br} 9 \mathrm{MeV}$ were carried out at CMAM-UAM facility. Moreover, with the goal of having a more complete set of irradiation at low fluences, a new group of rutile plates were irradiated with $\mathrm{Br}$ $13 \mathrm{MeV}$ at CMAM-UAM. The irradiations were performed under normal incidence at room temperature with fluences in the range of $6 \times 10^{12} \mathrm{~cm}^{-2}$ to $1.5 \times 10^{14} \mathrm{~cm}^{-2}$. Electronic and nuclear stopping powers obtained from SRIM 2003 simulations are plotted in Fig. 2. Electronic stopping powers $\left(S_{\mathrm{e}}\right)$ at the surface of $\mathrm{TiO}_{2}$ are: $5.3 \mathrm{keV} / \mathrm{nm}$ for $9 \mathrm{MeV} \mathrm{Br}$ ions, $6.9 \mathrm{keV} / \mathrm{nm}$ for $13 \mathrm{MeV} \mathrm{Br}$ ions and

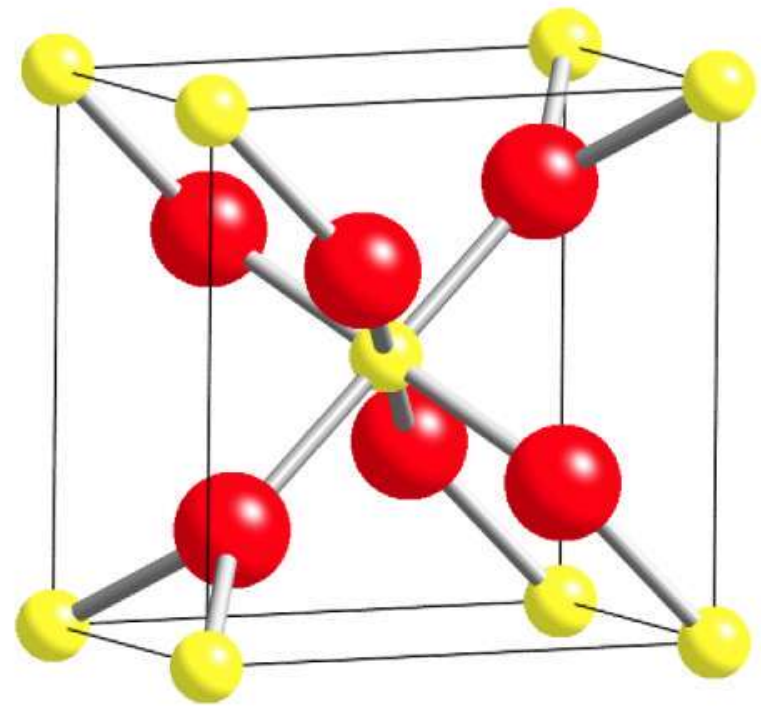

Fig. 1. A ball and stick model of the rutile structure illustrating the unit cell of the lattice. The Ti atoms (small balls) occupy the corners of the tetragonal structure, while the $\mathrm{O}$ atoms (big balls) appear inside the unit cell. 


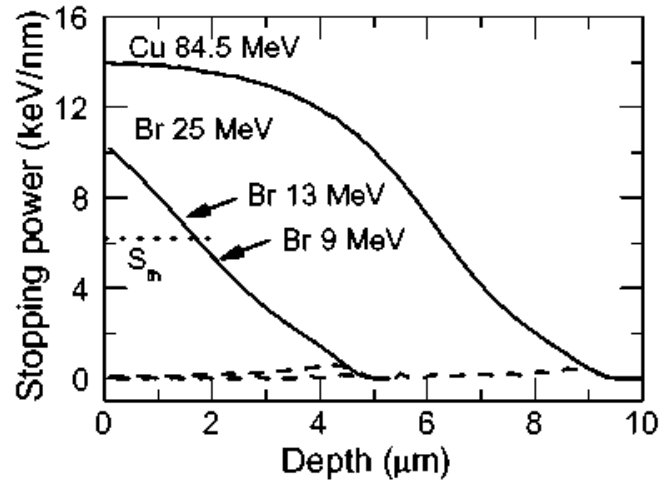

Fig 2. Electronic (solid) and nuclear (dashed) curves stopping power as a function of depth for irradiation of $\mathrm{TiO}_{2}$ with $\mathrm{Br}$ ions at $25 \mathrm{MeV}$ and $\mathrm{Cu}$ ions at $84.5 \mathrm{MeV}$. The arrows indicate the ion energy (in MeV) at different depths. The horizontal dotted line represents the threshold stopping power for $\mathrm{TiO}_{2}\left(S_{\mathrm{th}}=6.2 \mathrm{keV} / \mathrm{nm}\right)$.

$10.2 \mathrm{keV} / \mathrm{nm}$ for $25 \mathrm{MeV} \mathrm{Br}$ ions (indicated with arrows in Fig. 2). For comparison, the stopping powers corresponding to $84.5 \mathrm{MeV}$ $\mathrm{Cu}$ ions are also indicated in Fig. 2. The threshold stopping power $\left(S_{\mathrm{th}}\right)$ is indicated by the horizontal dotted line. Note that ions with stopping power above this value $\left(S_{e}>S_{t h}\right)$ produce single amorphous tracks $\left(S_{\text {th }}-6.2 \mathrm{keV} / \mathrm{nm}\right)$ for rutile $[14,17,18]$ ).

The created disorder was studied by channeling RBS experiments (RBS/C) with ${ }^{4} \mathrm{He}^{+}$ions at $3 \mathrm{MeV}$ at CMAM-UAM. They provide the damage fraction at the surface of rutile samples as a function of fluence for irradiations with $\mathrm{Br}$ ions at 9 and $13 \mathrm{MeV}$. Fig. 3 shows the results plotted as typical Avrami's curves [26]. Lattice disorder is progressively introduced even for fluences below $10^{13} \mathrm{~cm}^{-2}$. Note that these energies correspond to electronic stopping powers below and above the amorphization threshold $\left(S_{t h}\right)$. respectively, see Fig. 2 . Thus, irradiations with $\mathrm{Br}$ ions at $13 \mathrm{MeV}$ produce single ion tracks. When tracks fully overlap an amorphous layer is formed. This situation is detected by RBS/C experiments and corresponds to a disorder fraction of 1 . On the other hand, sub-threshold irradiations with $\mathrm{Br}$ ions at $9 \mathrm{MeV}$ can not produce single ion tracks. The damage detected by RBS/C experiments is originated by electronic excitation mechanisms that yields defect-formation. This damage presents a cumulative character that can even fully amorphize a layer when the fluence is high enough. Unfortunately, the maximum fluence in our experiments was too low to reach fully amorphization by irradiation with $\mathrm{Br}$ ions at $9 \mathrm{MeV}$. However, the trend clearly indicates that we could reach a disorder fraction of 1 (amorphization) at a fluence about $10^{14} \mathrm{~cm}^{-2}$.

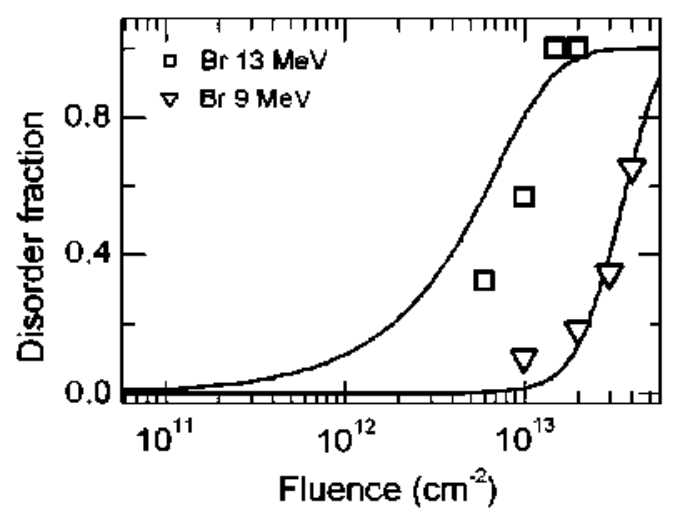

Fig. 3. Disorder fraction at the surface of rutile (100) samples irradiated with $\mathrm{Br}$ ions at $9 \mathrm{MeV}$ (inverted triangles) and $13 \mathrm{MeV}$ (squares). Both sets of samples were irradiated at CMAM-UAM. The solid lines are obtained with the exciton model (and the MonteCarlo algorithm).
The structure of the surface damage layer was investigated by optical reflectivity and $\mathrm{RBS} / \mathrm{C}$ [17]. Reflectivity data of rutile samples irradiated with $1.5 \times 10^{14} \mathrm{~cm}^{-2} \mathrm{Br}$ jons at $13 \mathrm{MeV}$ indicate that the refractive index changes significantly as an effect of the irradiation. This can be explained by the existence of a well-defined thin layer at the surface, which has a different refractive index (or density) than the underlying substrate. RBS/C experiments correspondingly indicate that the refractive index change occurs in an amorphized region. From the channelling data the density of the amorphous $\mathrm{TiO}_{2}$ can be calculated by using the thickness measured by reflectivity measurements. From the experiments a density of $3.6 \mathrm{~g} / \mathrm{cm}^{3}$ is obtained for amorphous $\mathrm{TiO}_{2}$. We have used this value to analyze the reflectivity measurements of samples irradiated with 13 and $25 \mathrm{MeV}$ using the 2 wave approximations for thin films at normal incidence [27]. Moreover, analysis of RBS/C experiments on $\mathrm{Br} 13$ and $25 \mathrm{MeV}$ irradiated samples has been carried out to determine the thickness of the amorphous layer. In this way, we have obtained the thickness of the amorphous layers formed as a consequence of ion irradiation (Fig. 4). For comparison we have included in Fig. 4 data from Ref. [18] on amorphous layers formed by irradiation with $\mathrm{Cu}$ ions at 84.5 MeV. In this case the thickness of the amorphous layers was determined by chemical etching in HF after irradiation. As shown in Fig. 4, the thickness of the amorphous layers raises abruptly beyond a fluence that roughly corresponds to a situation of full overlap of tracks. At high fluences the amorphous thickness tends to saturate at a depth significantly smaller than the ion implantation range. The saturation depth corresponds to a low electronic stopping power (see Fig. 2). For irradiations with $\mathrm{Cu}$ ions at $84.5 \mathrm{MeV}$ the maximum amorphous depth starts to saturate at $6 \mu \mathrm{m}$ and it seems that it will hardly reach $8 \mu \mathrm{m}$ at very high fluence. From Fig. 2, one gets a stopping power around $2 \mathrm{keV} / \mathrm{nm}$ for a depth of about $8 \mu \mathrm{m}$. It appears that the amorphization rate depends superlinearly on the stopping power and that values of $S_{e}$ as low as $2 \mathrm{keV} / \mathrm{nm}$ are not very efficient for amorphization. Furthermore, it is evident that the amorphization process is mainly due to electronic excitation mechanisms, being the nuclear effects of minor importance at the fluences employed in this work

The data illustrated in Fig. 4 clearly indicate that the thickness of such an amorphous layer increases with fluence, closely resembling the situation found for $\mathrm{LiNbO}_{3}$. In particular, the behaviour found in sub-threshold conditions (irradiation with $\mathrm{Br}$ at $9 \mathrm{MeV}$

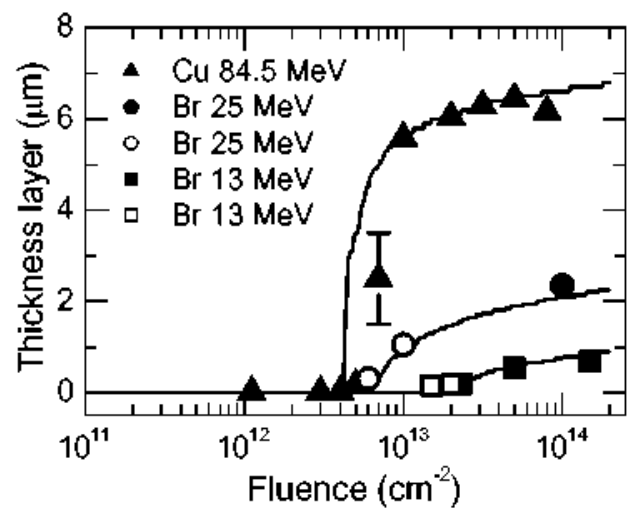

Fig 4. Thickness of the amorphous layer obtained after irradiation of rutile $\left(\begin{array}{lll}1 & 0 & 0\end{array}\right)$ samples with $\mathrm{Br}$ ions at $13,25 \mathrm{MeV}$ and $\mathrm{Cu}$ ions at $84.5 \mathrm{MeV}$. The thickness of the amorphous layer was determined by different methods; in the case of $\mathrm{Br} 25 \mathrm{MeV}$, by means of reflectivity (solid circles) and RBS/C measurements (open circles). In the case of $\mathrm{Br} 13 \mathrm{MeV}, \mathrm{RBS} / \mathrm{C}$ measurements for both, samples irradiated at CMAMUAM (open squares) and at Uppsala Universicy (solid squares). In the case of Cu ions, selective etching (data taken from Ref. [18]). The solid lines are obtained with the exciton model (and the Montecarlo algorithm). 
in Fig. 3) is not consistent with a thermal spike model and provides a definite proof for the cumulacive character of the damage. This suggests that an exciton model $[6,7]$ may be appropriate to describe the damage process.

\subsection{Analysis of the dato with the exciton model}

The exciton model previously developed $[6,7,28]$ has been now applied to explain the data described above. As for $\mathrm{LiNbO}_{3}$ the model is mostly phenomenological and is pending of a more complete physical understanding of electron and hole dynamics and exciton generation and decay. It is based on the synergy of nonradiative exciton decay and the generated thermal spike. The non-radiative exciton decay leads to point defect-formation but it is only possible thanks to the energy provided by the thermal spike. One can mathematically describe the exciton decay $\left(d N_{\mathrm{x}}\right)$ dt) as follows:

$\frac{d N_{X}}{d t}=-N_{X}(r, z, t)\left[v_{0} \exp \left(-\frac{\varepsilon}{k_{B} T(r, z, t)}\right)+\frac{1}{\tau}\right]$

where $v_{0}$ is a frequency factor, $\tau$ the radiative lifetime and $T(z, r, t)$ stands for the evolution of the temperature profile in the spike. Both profiles, the thermal spike $T(z, r, t)$ and the exciton spike $N_{x}(z, r, t)$ can be approximated by a Gaussian shape with a width $\mathfrak{a}_{0}$ as inferred from the thermal spike models [29]. The first term on the right corresponds to the non-radiative exciton decay and it is activated when energy from the thermal spike is available to surpass the energy barrier $\varepsilon$. The second term on the right describes the radiative exciton decay. Solving Eq. (1) allows one to obtain the concentration of defects $\left(\boldsymbol{n}_{v}\right)$ produced by an incoming ion (see [7] for details)

$\mathrm{n}_{v}(\mathrm{r}, z)=\int_{0}^{\infty} d t v_{0} N_{X}(r, z, t) \exp \left(-\frac{\varepsilon}{k_{\mathrm{B}} T(r, z, t)}\right)$

Another important point is that the model assumes that amorphization takes place when a critical defect concentration is reached, i.e., amorphization occurs as a defect driven transition whenever the stopping power of the ion exceeds the threshold value $\left(S_{\mathrm{th}}\right)$. Note that the model does not invoke melting as a necessary process for amorphization (as it is assumed in the thermal spike model), although, it is not excluded either.

We have developed a MonteCarlo approach to account for the amount and morphology of the damage produced by swift-ion irradiation. The approach uses a three dimensional cell structure on which jons hit randomly. The passage of every ion produces certain number of defects, $n_{v}(r, z)$, as given by Eq. (2). The cumulative character of the damage is considered by summing the contributions of two consecutive ions to every cell in the structure. If the defect concentration of one cell reaches the critical value that cell is assumed to become amorphous. In this way, both the Avrami's curves and the amorphous depth experiments can be simulated. Details of the MonteCarlo approach will be given elsewhere.

In order to simulate the experiments presented in Figs. 3 and 4, we have preliminarily fixed all but one parameter, the energy barrier $\varepsilon$. The values taken for the other parameters are justified as follows. The radiative lifetime $(\tau)$ is not very relevant as long as it is much longer than the spike duration. The gaussian width $a_{0}$ is assumed to be $4.5 \mathrm{~nm}$ as for $\mathrm{LiNbO}_{3}$ [7], $\mathrm{SiO}_{2}$ and other oxides [29]. The threshold stopping power $S_{\text {th }}$ is taken from $[14,17,18]$ to be $6.2 \mathrm{keV} / \mathrm{nm}$. After performing systematically many simulations the optimum value found for $\varepsilon$ is $2.0 \mathrm{eV}$. It is very remarkable that reasonable agreement is obtained for all the experiments presented in Figs. 3 and 4 with this choice of parameters. Note that the experiments span over a large range of conditions. Fig. 2 presents experiments mainly focused on low fluence to determine the disorder fraction at the surface, whereas Fig. 3 considers mainly experiments with strong track overlap to obtain amorphous layers. Irradiations with $\mathrm{Br}$ ions at $9 \mathrm{MeV}$ occur in subthreshold conditions, whereas all the other irradiations take place beyond threshold. We have used experiments from different sources and in addition several measurement techniques were employed: RBS/C, reflectivity and selective chemical etching. Despite this variety of experiments the model shows that it can reproduce fairly well the experimental data. In particular, the model reproduces the disorder fractions obtained at the surface for irradiations with $\mathrm{Br}$ ions at $13 \mathrm{MeV}$ (Fig. 2) and simultaneously, reproduces reasonably well the results obtained when irradiating at $9 \mathrm{MeV}$. Note that the thermal spike model fails to predict sub-threshold experiments such as those at $9 \mathrm{MeV}$. In addition, the thickness of the amorphous layer obtained by different techniques and research groups is surprisingly well reproduced.

Since such a variety of results has been reproduced, one may think that the model has an important value as a predictive tool, which is actually the goal behind the development of the model. Nevertheless, although the model seems applicable to a new material other than $\mathrm{LiNbO}_{3}$, we must be cautious with these preliminary results. Firstly, a better choice of parameters could be obtained allowing $a_{0}$ to change instead of fixing it based on the values obtained for other materials. Secondly, some aspects are missing in the model itself, as for example, an appropriate algorithm to obtain the initial exciton concentration or an algorithm to take into account the velocity effect.

\section{Summary and perspectives}

Alkali halides provide the reference system to discuss exciton models. From the extensive work on these materials some genera] guidelines have been put forward. Assuming the fulfilment of those premises, some preliminary irradiation data obtained on $\mathrm{TiO}_{2}$ have, indeed, shown that uniform amorphous layers are produced at moderate fluences as a result from full track overlapping. The data closely resemble those obtained for $\mathrm{LiNbO}_{3}$ and have been reasonably well described with the exciton model formalism. In addition, with the same parameters the model reproduces experiments obtained in sub-threshold conditions irradiating with $\mathrm{Br}$ jons at $9 \mathrm{MeV}$. Such experiments can not be explained in the framework of the thermal spike model. Although additional work must be carried out (both experimentally and theoretically), this suggests $\mathrm{TiO}_{2}$ as a new example of the applicability of the exciton model and suggests that it may be of general value for many oxide materials.

\section{References}

[1] D. Lesueur, A. Dunlop, Radiat. EIf. Def. Sol. 126 (1993) 163.

[2] M. Toulemonde, Ch. Dufour, A. Meftah, E. Paumier, Nucl. Instrum. Meth. Phys. Res. B 166-167 (2000) 903.

[3] M. Toulemonde, C. Trautmann, E. Balanzat, K. Hjort, A. Weidinger, Nucl. Instrum. Meth. Phys. Res. B 216 (2004) 1.

[4] A. Meftah, J.M. Costantini, N. Khalfaoui, 5. Boudjadar, J.P. Stoquert, F. Studer, M. Toulemonde, Nucl. Instrum. Methods Phys. Res. B 237 (2005) 563.

[5] G. Szenes, Phys. Rev. B 60 (1999) 3140.

[6] F. Agulló-lópez, A. Mendez, G. García, J. Olivales, J.M. Cabrera, Plıys. Rev, B 74 (2006) 174109

[7] A. Rivera, A. Méndez, G. García, J. Olivares, J.M. Cabrera, F. Agulló-lópez, J, Lumin. 128 (2008) 703

[8] N. Itoh, Nucl. Instrum. Meth. Phys. Res. B 135 (1998) 175.

[9] F. Agulló-López, R.C. Catlow. P.D. Townsend, Point Defects in Materials, Academic Press, 1984.

[10] F. Agulló-López, P.D. Townsend, Solid St. Commun. 33 (1980) 449.

11] P.D. Townsend, F. Agulló-Lopez, J. Phys. (Paris), Colloq. C6 41 (1980) 279.

[12] N. Itoh, A.M. Stoneham, Materials Modification by Electronic Excitation, Cambridge Universicy Press, 2001.

[13] M. Langlet, M. Burgos, C. Coutier, C. Jimenez, C. Morant, M. Manso, J. Sol-Gel Sci. Technol, 22 (2001) 139.

|14| K. Awazu, M. Fujimaki, Y. Olıki, T. Komatsubara, Rad. Meas. 40 (2005) 722. 
|15| K. Awazu, X. Wang, M. Fujinkaki, T. Konatsubara, T. Ikeda, Y. Ollki, J. Appl. Phys. $100(2006) 044308$.

|16| N. Ishikawa, 5. Yamamoto, Y. Chimi, Nucl. Instrum, Metl. Phys. Res. B 250 (2006) 250 .

[17| J. Jensen, M. Skupiński, K. Hjort, R. Sanz, Nucl. Instrum. Meth. Phys. Res. 266 (2008) 3113.

[18] K. Nomura, T. Nakanishi, Y. Nagasawa, Y. Ohki, K. Awazu, M. Fujinami, N. Kobayashi, S. Ishii, K. Shima, Phys. Rev. B 68 (2003) 064106.

[19] V. Murk, Materials Science Forum 239-241 (1997) 537.

|20| J.G. Traylor, H.G. Snith, R.M. Nicklow, M.K. Wilkinson, Phys, Rev, B 3457 (1971).
[21] FA. Grant, Reviews of Modern Physics 3 (1959) 646

[22] LG.J. de Haart, G. Blasse, J. Solid State Chem. 61 (1986) 135.

[23] H. Tang. H. Berger, P.E. Schmid, F, Levy. Solid State Commun. 87 (1993) 847.

[24] A. Amtout, R. Leonelli, Phys. Rev. 51 (1995) 6842.

[25] I. Sildos, A. Suisalu. J. AArik, T. Sekiya, 5. Kurita, J. Lumin. 87-89 (2000) 290.

[26] A. García-Navarro, F. Agulló-lópez, M. Bianconi, J. Olivares, G. García, J. Appl. Phys. 101 (2007) 83506.

[27] E. Heclit, Optics, second ed., Addison Wesley, Reading Massachussets, 1987.

[28] A. Rivera, J. Olivares, G. García, J.M. Cabrera, F. Agulló-Rueda, F. Agulló-López, Physica Status Solidi A 206 (2009) 1109.

[29] G. Szenes, Nucl. Instrum. Meth. Phys. Res. B 122 (1997) 530. 